\title{
The synthesis of SSZ-13 with mixed template and it's catalytic performance for methanol to olefins reaction
}

\author{
Yunshui Deng ${ }^{1, a}$, Yuan $\mathrm{Li}^{1, \mathrm{~b}^{\star}}, \mathrm{Yu} \mathrm{Su}{ }^{1, \mathrm{c}}$ \\ ${ }^{1}$ Tianjin Polytechnic University, school of environmental and chemical engineering \\ Tianjin, 300387, China \\ a email:xsdllqn1204@yeah.net, ${ }^{\mathrm{b}}$ email: liyuan@tjpu.edu.cn, ${ }^{\mathrm{c}}$ email: tjusuyu@126.com
}

Keywords: CHA; SSZ-13; MTO; mixed template; BTMA ${ }^{+}$

\begin{abstract}
In order to reduce the cost, a mixed template of $\mathrm{BTMA}^{+}$and $\mathrm{TMADA}^{+}$was used to synthesize SSZ-13 zeolite. The effects of the compositions of the mixed template on structure and morphology were characterized by XRD, SEM. The catalytic performance of prepared SSZ-13 was investigated in a fixed-bed reactor at $450{ }^{\circ} \mathrm{C}$ for the methanol-to-olefin reaction ,the weight hourly space velocity (WHSV) was $1.75 \mathrm{~h}^{-1}$. The results show that cubic SSZ-13 crystals with good crystallinity and small size distribution (the average particle size of $0.3 \mathrm{um}$ ) was synthesized with the $\mathrm{n}\left(\mathrm{TMADA}^{+}\right) / \mathrm{n}\left(\mathrm{TMADA}^{+}+\mathrm{BTMA}^{+}\right)$is 0.3 . In the methanol-to-olefin reaction, The yield of olefin can reaches $88 \%$, and the lifetime of the catalyst is above $200 \mathrm{~min}$.
\end{abstract}

\section{Introduction}

Zeolites are microporous crystalline materials that have found widespread commercial applications as catalysts, ion-exchangers and adsorbers with it's very large specific surface area and perfect catalytic activity [1]. SSZ-13 zeolite is a microporous molecular sieve, which have chabazite (CHA) topology. The basic unit of the aluminosilicate framework structure for the SSZ-13 Zeolite is a 8-ring, which can form through the connection of oxygen bridge units composed of 4-rings and 6-rings. SSZ-13 zeolite was first synthesized by Stacey I. Zones using hydrothermal method in the 80 s of 20th century[2]. The large specific surface area and Unique framework structure make it has excellent performance in gas mixture separation and catalysis field[3,4]. Falconer et al. reported that the SSZ-13 zeolite membrane could separate light-gas mixtures of $\mathrm{CO} / \mathrm{N}_{2}, \mathrm{CO}_{2} / \mathrm{CH}_{4}, \mathrm{H}_{2} / \mathrm{CH}_{4}$, and $\mathrm{H}_{2} / \mathrm{n}-\mathrm{C}_{4} \mathrm{H}_{10}$ with notably high selectivity[3]. Hasegawa et al. found that the CHA-type zeolite membrane exhibited excellent dehydration performance for water/alcohol mixtures[5,6]. J.F Haw et al. reported that the CHA topology have potential industrial application, with highly selective catalysis in the methanol-to-olefin (MTO) process [7] .

As the SSZ-13 zeolite has been synthesized using N,N,N-trimethyl-1-adamant ammonium $\left(\mathrm{TMADA}^{+}\right)$as structure-directing agent (SDA) $[8,9,10]$, this SDA is too costly to entirely utilize it's capacity. As synthesized zeolite without or with cheaper SDA is more competitive in commercial field[11,12], a lots efforts have been applied to generating improved methods for the synthesis CHA-type zeolite by using a small amount of TMADA ${ }^{+}$or other unexpensive SDAs. Very recently, there are a few of papers published related with the synthesis of SSZ-13, in which benzyl trimethyl ammonium cation $\left(\mathrm{BTMA}^{+}\right)$instead of a typical SDA[13,14]. We have tried many times to synthesis SSZ-13 only used BTMA ${ }^{+}$as SDA and result with an impure phase of CHA zeolite. So we can infer that the compositions range of the raw materials for SSZ-13 is very narrow or the reaction condition is very difficulty to reach. But $\mathrm{BTMA}^{+}$is much cheaper than $\mathrm{TMADA}^{+}$in industrial, and the structure of $\mathrm{BTMA}^{+}$is very similar to that of $\mathrm{TMADA}^{+}$, therefore, in order to reduce the cost, a mixture template of $\mathrm{BTMA}^{+}$and $\mathrm{TMADA}^{+}$were used to synthesize SSZ-13 zeolite in this paper. The effects of the compositions of the mixed template on SSZ-13 and the catalytic performance for methanol-to-olefins reaction were discussed in detail.

\section{Experimental}




\section{Synthesis of SSZ-13 molecular sieve.}

SSZ-13 molecular sieves were hydrothermally synthesized with a mixture of BTMAOH and TMADAOH as the template agent. Aluminum sulfate, silica sol were used as the sources of $\mathrm{Al}$ and $\mathrm{Si}$, respectively. The molar composition of synthesis solution was 1.0 $\mathrm{Al}_{2} \mathrm{O}_{3}: 80 \quad \mathrm{SiO}_{2}:(\mathrm{x}): 4 \mathrm{Na}_{2} \mathrm{O}$ TMADAOH : (20-x) BTMAOH : $300 \mathrm{H}_{2} \mathrm{O}(\mathrm{x}=2,4,6,10)$. In detail, weighted aluminum sulfate was dissolved into dis-tilled water under vigorous stirring for $0.5 \mathrm{~h}$ at room temperature, and silica sol were added in drop. After the synthesis solution was stirred for $2 \mathrm{~h}$, the template agent was added and it was continually stirred for another $2 \mathrm{~h}$, then the synthesis gel was transferred into a stainless-steel autoclave and was heated at $160{ }^{\circ} \mathrm{C}$ for $4 \mathrm{~d}$. The solid product was separated by centrifugation, washed with

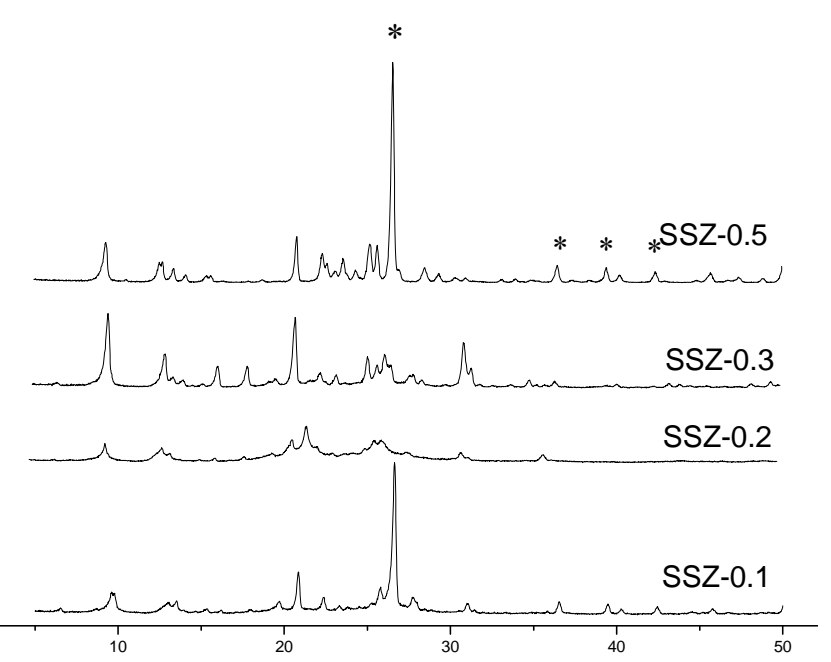

Fig. 1. XRD patterns of prepared samples (*Standing for the phase of Quartz) distilled water several times, and dried overnight at $120{ }^{\circ} \mathrm{C}$. Finally, the catalyst sample was calcined at $600{ }^{\circ} \mathrm{C}$ for $6 \mathrm{~h}$ to remove organic template.

\section{Characterization of sample.}

The structure phase of the sample was analyzed by powder X-ray diffraction (XRD) on a D/MAX-2500 Diffractometer with $\mathrm{Cu} \mathrm{K} \alpha$ radiation $(\lambda=1.5406 \AA)$ and a graphite monochromator at $40 \mathrm{kV}, 150 \mathrm{~mA}$.

\section{Catalytic activity evaluation.}

The performance of the catalyst for the MTO reaction was tested in a fixed-bed stainless steel micro-reactor $(\theta, 10 \mathrm{~mm} \times 380 \mathrm{~mm}) .1 .0 \mathrm{~g}$ catalyst was loaded and pretreated in $120 \mathrm{ml} / \mathrm{min}$ dry nitrogen at $500{ }^{\circ} \mathrm{C}$ for $30 \mathrm{~min}$. After the temperature of reactor was decreased to $450{ }^{\circ} \mathrm{C}$, methanol and $\mathrm{H}_{2} \mathrm{O}(1 / 4$, mol) was fed by syringe pump to the reactor, and the weight hourly space velocity (WHSV) was $1.75 \mathrm{~h}^{-1}$. The products were analyzed by an gas chromatograph (GC9800) equipped with an HP-PLOT-Q column and flame ionization detector (FID).

\section{Results and discussion}

\section{Effect of template composition on the structure of sample prepared.}

In order to determine the effect of the mixed template compositions on the physicochemical properties and the catalytic performance of the sample prepared, herein, a set of the SSZ-13 samples were prepared with different template compositions under same other parameters. The preparation conditions for SSZ-13 samples and their structure phase are shown in Table 1.

Table 1 The gel compositions and structure phases of prepared samples

\begin{tabular}{|c|c|c|c|c|c|c|c|}
\hline \multirow{2}{*}{ Sample } & \multicolumn{6}{|c|}{ Composition of synthesis gel $[\mathrm{mol}]$} & \multirow{2}{*}{ Structure phase } \\
\hline & $\mathrm{Na}_{2} \mathrm{O}$ & $\mathrm{Al}_{2} \mathrm{O}_{3}$ & $\mathrm{SiO}_{2}$ & $\mathrm{TMADA}^{+}$ & BTMA $^{+}$ & $\mathrm{n}\left(\mathrm{TMADA}^{+}\right) / \mathrm{n}\left(\mathrm{TMADA}^{+}+\mathrm{BTMA}^{+}\right)$ & \\
\hline \multirow{2}{*}{ SSZ-0.1 } & \multirow{2}{*}{4.0} & \multirow{2}{*}{1.0} & \multirow{2}{*}{80.0} & \multirow{2}{*}{2} & \multirow{2}{*}{18} & \multirow{2}{*}{ (2) } & SSZ-13+Qua \\
\hline & & & & & & & rtz \\
\hline SSZ-0.2 & 4.0 & 1.0 & 80.0 & 4 & 16 & 0.2 & $\begin{array}{l}\text { SSZ-13(Trac } \\
\text { e) }\end{array}$ \\
\hline SSZ-0.3 & 4.0 & 1.0 & 80.0 & 6 & 14 & 0.3 & SSZ-13 \\
\hline SSZ-0.5 & 4.0 & 1.0 & 80.0 & 10 & 10 & 0.5 & $\begin{array}{l}\text { SSZ-13+Qua } \\
\text { rtz }\end{array}$ \\
\hline
\end{tabular}

Fig. 1 shows the XRD patterns of SSZ samples prepared with different template compositions. For the SSZ-0.3 samples prepared by using $\mathrm{BTMA}^{+}$template with $\mathrm{TMADA}^{+}$, the typical XRD patterns of CHA-structure SSZ-13 can be observed in their XRD patterns, where the intensity and 
position of each peak match well with that reported for SSZ-13 materials without any presence of impurity phases [2]. Comparing with the SSZ-0.3 sample, besides the diffraction peaks of SSZ-13, SSZ-0.1 and SSZ-0.5 appear some diffraction peaks of Quartz at $2 \theta$ of $26.64^{\circ}, 36.54^{\circ}, 42.42^{\circ}$, and $45.82^{\circ}$. Based on the XRD results, it can be concluded that the pure phase SSZ-13 molecular sieve can be synthesized when the molar ratio of $n\left(\mathrm{TMADA}^{+}\right) / \mathrm{n}\left(\mathrm{TMADA}^{+}+\mathrm{BTMA}^{+}\right)$is 0.3 .

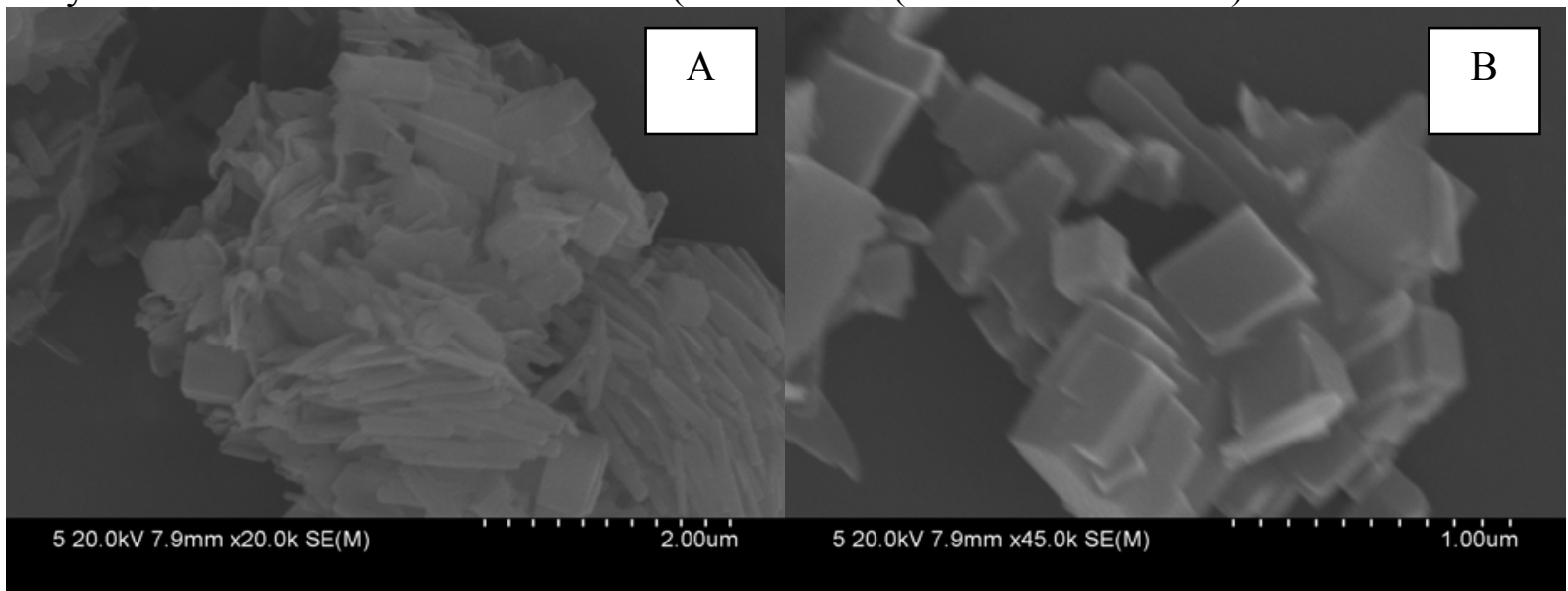

Fig.2. SEM images of prepared samples of (A) SSZ-0.1 and(B) SSZ-0.3.

Fig. 2 shows the SEM images of the SSZ samples. SSZ-0.1 sample prepared with mixed template agent exhibits an image of stripy-like quartz crystals as the main phase coexist with cubic-like SSZ-13 crystals (Fig. 2(A)). SSZ-0.3 sample shows cube type SSZ-13 crystals (Fig. 2(B)) with an average particle size of $0.3 \mathrm{um}$ and size distribution in the range of $0.2-0.5 \mathrm{um}$ and some of them are aggregated. These results show that the morphology of SSZ-13 molecular sieves is depended on the composition of mixture template largely. When the $\mathrm{n}\left(\mathrm{TMADA}^{+} / \mathrm{n}\left(\mathrm{TMADA}^{+}+\mathrm{BTMA}^{+}\right)\right.$is 0.3 was used, cubic SSZ-13 crystals with good Crystallinity and small size distribution can be synthesized.

\section{Catalytic performances of prepared SSZ-0.3 catalysts.}

Catalytic performances of SSZ-0.3 catalysts for the methanol-to-olefins (MTO) reaction were tested and the product distribution with different operation lifetime are shown in Fig 3. It can be seen that high selectivity of light olefins was obtained in the initial time and increased with the decreasing of propane content selectivity. As we know, the strong acid site is the source of hydrogen transfer reactions which can transfer olefins into alkanes[15]. After the initial period of reaction, the coking will formed gradually, as a result, the strong acidity will reduce. Therefore, the reaction from propylene to propane decrease, and the yield of olefins increase with time on stream. It was also found that the selectivity of methane increased with time. The good catalytic performance and a long lifetime about 200 minutes in the MTO means SSZ-13 zeolite is a potential catalyst for MTO.
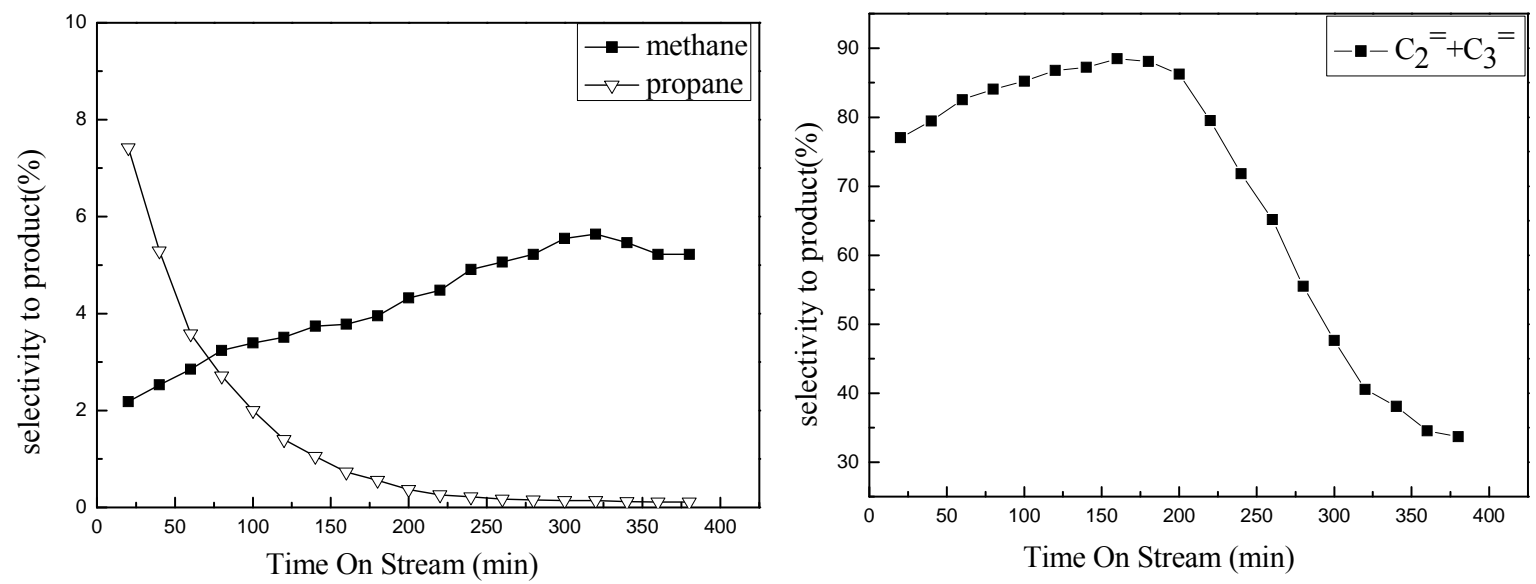

Fig 3 the Product distribution in the MTO reaction over the SSZ-0.3 catalyst

Reaction condition: $\mathrm{CH}_{3} \mathrm{OH} / \mathrm{H}_{2} \mathrm{O}(1 / 4, \mathrm{~mol}), \mathrm{N}_{2}$ of $120 \mathrm{ml} / \mathrm{min}$, WHSV of $1.75 \mathrm{~h}^{-1}, 450^{\circ} \mathrm{C}$ 


\section{Conclusions}

A mixed template of BTMAOH and TMADAOH was used to synthesis SSZ-13 zeolite. The pure phase can be obtained when the ratio of $n\left(\mathrm{TMADA}^{+} / \mathrm{n}\left(\mathrm{TMADA}^{+}+\mathrm{BTMA}^{+}\right)\right.$is 0.3 . On the other ratio, impure phase of quartz with stripy-like type, as the main phase, will exist. The performance and the stability of SSZ-13 for the MTO reaction were tested. The yield of olefin can reaches $88 \%$, and the lifetime of the catalyst is above $200 \mathrm{~min}$. So SSZ-13 zeolite is a potential catalyst for MTO.

\section{References}

[1] A. Corma, Chem. Rev. 95 (1995) 559.

[2] Zones, S. I. US patent 4,544,538 (1985)

[3] H. Kalipcilar, T.C. Bowen, R.D. Noble, J.L. Falconer, Chem. Mater. 14 (2002) 3458.

[4] M. Stcker, Micropor. Mesopor. Mater. 29 (1999) 3.

[5] Y. Hasegawa, H. Hotta, K. Sato, T. Nagase, F. Mizukami, J. Membr. Sci. 347 (2010) 193.

[6] Y. Hasegawa, C. Abe, M. Nishioka, K. Sato, T. Nagase, . J. Membr. Sci.364 (2010) 318.

[7] J.F. Haw, W. Song, D.M. Marcus, J.B. Nicholas, Acc. Chem. Res. 36 (2003) 317.

[8]Yufeng Wang, Yuan Li ,Enqi Tang,International Journal of Chemistry,2010,2:128

[9]Wang Yu-feng,Li Yuan,Tang En-qi,Petroleum Processing and Petrochemicals,2010 Vol. 41 (2):11 [10]WangYu-feng,Li Yuan,Tang En-qi,Journal of Tianjin Polytechnic University,Vol.29 No.1(2010):64

[11]Liu Zh M, Sun Ch L, Wang G W, Wang Q X, Cai G Y. Fuel Process Technol, 2000, 62: 161

[12]Li Yuan,Dou Guosheng,Ma Gangfeng,Du Qingkun,Shen Yixin,Li Yong,CN 102134081 A (2011).

[13]M. Itakura, T. Inoue, A. Takahashi, T. Fujitani, Y. Oumi, T. Sano, Chem. Lett. 37 (2008) 908.

[14] M. Itakura, I. Goto, A. Takahashi, T. Fujitani,„Micropor. Mesopor. Mater. 144 (2011) 91.

[15]Q J Zhu, Junko N. K, Ryosuke O, Yoshihiro K ,et al .Micro .Meso. Mat.112 (2008) 153 